\title{
Are Serial CA 19-9 Kinetics Helpful in Predicting Survival in Patients with Advanced or Metastatic Pancreatic Cancer Treated with Gemcitabine and Cisplatin?
}

\author{
$\begin{array}{llll}\text { J. Stemmler } & \text { P. Stieber } & \text { A. M. Szymala } & \text { A. Schalhorn } \\ & \text { C. M. Schermuly } & \text { R. Wilkowski }\end{array}$

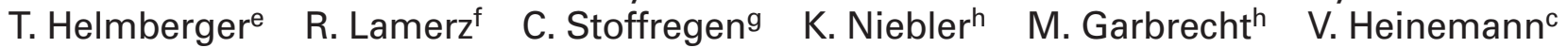 \\ ${ }^{a}$ Dept. of Oncology, Onkologische Klinik Bad Trissl, Oberaudorf, bept. of Clinical Chemistry, ${ }^{\mathrm{c}}$ Dept. of Internal Medicine III - Oncology, \\ ${ }^{\mathrm{d} D e p t . ~ o f ~ R a d i o t h e r a p y ~ a n d ~ R a d i o o n c o l o g y, ~ e ~ D e p t . ~ o f ~ R a d i o l o g y, ~}{ }^{\mathrm{f}}$ Dept. of Internal Medicine II, University of Munich, \\ gLilly Deutschland GmbH, Bad Homburg, hDept. of Internal Medicine / Oncology, Clinic München-Neuperlach, Germany
}

\section{Key Words}

CA 19-9 · Tumor marker · Combination therapy: gemcitabine, cisplatin . Pancreatic cancer

\section{Summary}

Background: Serial kinetics of serum CA 19-9 levels have been reported to reflect response and survival in patients with pancreatic cancer undergoing surgery, radiotherapy, and chemotherapy. We prospectively studied serial kinetics of serum CA 19-9 levels of patients with locally advanced or metastatic disease treated with gemcitabine and cisplatin. Patients and Methods: Enrolled in the study were 87 patients (female $/$ male $=26 / 61$; stage III/IV disease $=24 / 63$ ) Patients received gemcitabine $1,000 \mathrm{mg} / \mathrm{m}^{2}$ on days 1,8 , and 15 plus cisplatin $50 \mathrm{mg} / \mathrm{m}^{2}$ on days 1 and 15 , every 4 weeks. Serum samples were collected at the onset of chemotherapy and before the start of a new treatment cycle (day 28). Results: 77 of 87 patients $(88.5 \%)$ with initially elevated CA $19-9$ levels were included for evaluation. According to imaging criteria, 4 (5.2\%) achieved a complete remission and $11(14.3 \%)$ achieved partial remission, yielding an overall response rate of $19.5 \% .43(55.8 \%)$ patients were CA $19-9$ responders, defined by a $\geq 50 \%$ decrease in CA $19-9$ serum levels within 2 months after treatment initiation. Except for one, all patients who had responded by imaging criteria $(n=14)$ fulfilled the criterion of a CA 19-9 responder. Despite being characterized as non-responders by CT-imaging criteria (stable/progressive disease), 29 patients were classified as CA 19-9 responders (positive predictive value $32.5 \%$ ). Independent of the response evaluation by $\mathrm{CT}$, CA 19-9 responders survived significantly longer than CA 19-9 nonresponders ( 295 d; $95 \%$ Cl: $285-445$ vs. 174 d; $95 \%$ Cl: $134-198 ; p=$ 0.022). Conclusion: CA $19-9$ kinetics in serum serve as an early and reliable indicator of response and help to predict survival in patients with advanced pancreatic cancer receiving effective treatment with gemcitabine and cisplatin.

\section{Schlüsselwörter}

CA 19-9 - Tumormarker - Kombinationschemotherapie: Gemcitabin, Cisplatin · Pankreaskarzinom

\section{Zusammenfassung}

Hintergrund: Verlaufsmessungen von CA-19-9-Spiegeln haben eine Aussagekraft bezüglich der Ansprechrate und Überlebenszeit von Patienten die wegen eines Pankreaskarzinoms operiert, bestrahlt oder chemotherapiert werden. In der vorliegenden Arbeit wurden prospektiv CA-19-9-Spiegel von Patienten mit lokal fortgeschrittenem oder metastasiertem Pankreaskarzinom untersucht, die mit einer Kombinationschemotherapie bestehend aus Gemcitabin und Cisplatin behandelt wurden. Patienten und Methoden: Insgesamt wurden 87 Patienten $(\mathrm{m} / \mathrm{w}=26 / 61$; Stadium III/IV = 24/63) in die Studie eingeschlossen. Die Tumormarkerspiegel wurden unmittelbar vor Beginn der Chemotherapie, und im Verlauf vor jedem weiteren Chemotherapiezyklus bestimmt. Die Chemotherapie bestand aus Gemcitabine $1000 \mathrm{mg} / \mathrm{m}^{2}$ (Tag 1, 8, 15) und Cisplatin $50 \mathrm{mg} / \mathrm{m}^{2}$ (Tag 1, 15), und wurde an Tag 28 wiederholt. Ergebnisse: Von 87 eingebrachten Patienten hatten 77 initial erhöhte CA-19-9-Spiegel (88,5\%) und wurden daher weiter ausgewertet. Nach Bildgebungskriterien (CT-Befund) erreichten 4 Patienten eine komplette Remission $(5,2 \%)$ und $11(14,3 \%)$ eine partielle Remission, so dass eine Gesamtansprechrate von $19,5 \%$ resultiert. Von insgesamt 77 Patienten mit initial erhöhten CA-19-9-Spiegeln erfüllten $43(55,8 \%)$ das Kriterium eines "CA-19-9-Responders" (definiert als Abfall des CA 19-9 $\geq 50 \%$ innerhalb der ersten 2 Monate nach Beginn der Behandlung). Bis auf einen Patienten erfüllten alle Patienten die nach Bildgebungskriterien angesprochen hatten $(n=14)$ auch das Kriterium eines "CA19-9-Responders". Interessanterweise wurden unter den Patienten die nach Bildgebungskriterien nicht angesprochen hatten (SD/PD), dennoch 29 Patienten als "CA-19-9-Responder" klassifiziert. Unabhängig vom CT-Befund lebten "CA-19-9-Responder» signifikant länger als "CA-19-9-Non-Responder» (295 Tage; 95\%-Cl: 285-445 vs. 174 Tage; 95\%-Cl: 134-198; $p=0,022$ ). Schlussfolgerung: CA-19-9Spiegel im Verlauf einer Chemotherapie mit Gemcitabin und Cisplatin erlauben bei Patienten mit Pankreaskarzinom neben einer frühen Beurteilung des Ansprechens auf die Chemotherapie auch eine Prognose bezüglich der Überlebenszeit.

\begin{tabular}{ll}
\hline KARGER & $\odot 2003$ S. Karger GmbH, Freiburg \\
Fax +49 76145207 14 & Accessible online at: \\
$\begin{array}{l}\text { E-mail Information@Karger.de } \\
\text { www.karger.com }\end{array}$ & www.karger.com/onk \\
&
\end{tabular}




\section{Introduction}

Pancreatic cancer is currently the fifth most frequent tumorrelated cause of death. Unresectable pancreatic cancer has a dismal prognosis, and 5-year survival is generally less than 5\% [1]. Therapeutic efforts at tumor stages III and IV are essentially directed toward palliation, because a cure cannot be achieved in most patients. Since the introduction of gemcitabine, which is superior to 5-FU (5-fluorouracil) regarding clinical benefit, response, and survival, great efforts have been undertaken to evaluate the impact of chemotherapy by methods other than imaging of tumor volume [1-8].

Response evaluation by standard imaging procedures is particularly complicated by limited differentiation of tumor from normal surrounding tissue, which is partly explained by desmoplastic and local inflammatory reactions induced by the tumor [3]. One approach has been to measure clinical benefit response, which is a composite endpoint consisting of pain, analgesic consumption, performance status, and weight, but the value of this parameter as a convenient and reliable surrogate endpoint of response still remains debatable [9].

In search for a quick and objective response evaluation, CA 19-9 kinetics have been analyzed in patients undergoing chemotherapy of pancreatic carcinoma. CA 19-9 is a sialylated Lewis antigen known as a sensitive marker in pancreatic cancer [10-17]. Although it is generally agreed that tumor markers are inadequate screening tools for the diagnosis of cancer $[12,18]$, they may well serve to guide therapy of proven cancer disease. CA 19-9 has been used as a prognostic indicator of disease status during follow-up evaluations after surgery, radio-, or chemotherapy [17]. There is no agreement, however, to which extent CA 19-9 can be used as a surrogate endpoint for response evaluation during chemotherapy of advanced or metastatic disease. Moreover, a clear definition of CA 19-9 response has not been established. In previous studies CA 19-9 response was defined as a decrease from baseline ranging between 15 and $50 \%[16,19,20]$. The goal of this study was to evaluate the value of CA 19-9 kinetics as a response parameter complementary to conventional radiological imaging and to define its prognostic importance during intensive chemotherapy with gemcitabine and cisplatin.

\section{Patients and Methods}

\section{Patient Selection}

The current analysis includes the data of two previously published clinical trials $[6,8]$. Inclusion criteria for the present study were histologically or cytologically proven advanced or metastatic pancreatic cancer; bidimensionally measurable disease; relapsing disease or disease not responding to initial radiochemotherapy; Karnofsky performance status of $\geq 70 \%$; age 18-70 years; and anticipated survival of at least 12 weeks. In addition, cardiac, hepatic, renal, and hematological function had to be adequate. $\mathrm{Pa}$ tients were excluded for active infection; inadequate renal or cardiac function; and a history of a second malignancy other than resected basal cell and/or squamous cell carcinoma of the skin. All patients gave written in- formed consent, and the local ethics committee approved the treatment protocol.

\section{Treatment Regimen}

Initially 34 patients received a combination chemotherapy consisting of gemcitabine $1,000 \mathrm{mg} / \mathrm{m}^{2}$ on days 1,8 , and 15 , and cisplatin $50 \mathrm{mg} / \mathrm{m}^{2}$ on days 1 and 15 . To improve treatment tolerability and to reduce toxicity, the regimen was subsequently modified in a second trial by omitting the day- 8 gemcitabine dose in 43 patients. Treatment was administered in 4 week cycles, and continued until disease progression or occurrence of severe side effects.

\section{Baseline and Treatment Assessments}

Standard evaluation by history, physical examination, and routine laboratory tests was performed before each treatment. Imaging studies using computerized tomography (CT) were performed after every 2 cycles of treatment; only bidimensionally measurable lesions were used for these response evaluations. For all patients, tumor lesions were measured by CT within 14 days of entry into the study and subsequently after every 2 cycles of treatment. The criterion assessing the clinical response was the best response at any time during treatment and follow-up. Patient response was assessed by standard WHO criteria [21]. Drug administration, performance status, toxicity, and adverse events were recorded after every cycle of treatment. Toxicity was graded according to the National Cancer Institute Common Toxicity Criteria [22].

\section{Determination of CA 19-9 Serum Concentrations}

CA 19-9 serum concentrations were prospectively determined by an automated enzyme immunoassay based on the sandwich principle (Enzymun ${ }^{\circledR}$, Boehringer Mannheim, ES 700, Germany). Serum samples were routinely collected at the onset of chemotherapy and before the start of any new treatment cycle (day 28). CA 19-9 response was defined as a $\geq 50 \%$ decrease from pretreatment levels within 2 months after the start of treatment and was evaluated according to the criteria of Ishii et al. [16]. If another threshold was chosen for defining CA 19-9 response this is indicated in the tables.

To be considered evaluable for response, patients had to complete at least 2 cycles of chemotherapy and required elevated CA 19-9 levels above the normal range at baseline. According to previous studies evaluating CA 19-9 in healthy volunteers, a cut-off value of $32 \mathrm{U} / \mathrm{ml}$, reflecting the 95 th percentile, was used as the upper limit of the normal (ULN) range [18].

\section{Statistical Evaluation}

Survival times were measured from the date of the start of treatment to the date of death from any cause. The probability of survival was estimated by Kaplan-Meier analysis [23]. Differences between patient groups in survival and differences between other parameters were calculated using the log-rank or t-test. Changes in marker expression were compared with CT-scans. These changes were expressed in terms of sensitivity, specificity, and positive or negative predictive value. The following definitions apply: Sensitivity $=$ true positive $/($ true positive + false negative $) \times 100 \%$, Specificity $=$ true negative $/($ true negative + false positive $) \times 100 \%$, Positive predictive value $(\mathrm{PPV})=$ true positive $/($ true positive + false pos itive) $\times 100 \%$,

Negative predictive value $(\mathrm{NPV})=$ true negative $/$ (true negative + false negative) $\times 100 \%$.

\section{Results}

\section{Patient Characteristics}

Between September 1994 and January 2001, 87 patients, 61 males and 26 females, with advanced pancreatic cancer were 
Table 1. Patient characteristics

\begin{tabular}{ll}
\hline Patients enrolled, $\mathrm{n}$ & 87 \\
Male/female, $\mathrm{n}(\%)$ & $61 / 26(70.1 / 29.9)$ \\
Age, years & 59 \\
$\quad$ Median & $33-72$ \\
$\quad$ Range & \\
Karnofsky performance status, \% & 80 \\
$\quad$ Median & $70-100$ \\
$\quad$ Range & \\
Disease stage, n (\%) & $24(27.6)$ \\
$\quad$ Stage III (T1-4, N1, M0) & $63(72.4)$ \\
$\quad$ Stage IV (T1-4, N0-1, M1) & \\
\hline
\end{tabular}

Patients without elevated CA 19-9 levels $(n=10)$

Elevated bilirubin serum levels, $\mathrm{n}(\%)$
$\geq 1.1 \mathrm{mg} / \mathrm{dl}$
$4(40)$
$\geq 3.0 \mathrm{mg} / \mathrm{dl}$
0

Patients with elevated CA 19-9 levels $(n=77)$

Male/female, n (\%)

Disease stage, $\mathrm{n}(\%)$

Stage III (T1-4, N1, M0)

Stage IV (T1-4, N0-1, M1)

Median CA 19-9 (range), U/ml

Stage III disease (T1-4, N1, M0)

Stage IV disease (T1-4, N0-1, M1)

Elevated bilirubin serum levels, $\mathrm{n}(\%)$

$\geq 1.1 \mathrm{mg} / \mathrm{dl}$

$\geq 3.0 \mathrm{mg} / \mathrm{dl}^{\mathrm{a}}$

Liver metastases, $\mathrm{n}(\%)$

Prior therapy, n (\%)

No prior therapy

Surgery of curative intent

Surgery of palliative intent

Radio-/chemotherapy (5-fluorouracil-based), n (\%)

$16(20.8)$

61 (79.2)

$953(44-742,398)$

344 (53-5001)

$1,911(44-742,398)^{*}$

17 (22.1)

$7(9.1)$

$56(72.7)$

59 (76.6)

14 (18.2)

4 (5.2)

$5(6.5)$

* $\mathrm{p}<0.001$ for the difference between median CA 19-9 values of patients with disease stages III and IV.

${ }^{a}$ In 3 patients, bilirubin decreased to normal range after stent implantation.

recruited. The present analyses hereby included data of two previously published trials. Detailed informations about patient characteristics are shown in table 1.

\section{Response Evaluation by CT-Imaging and Survival}

Of the 77 patients evaluable for response, 4 (5.2\%) achieved a complete remission (CR) and $11(14.3 \%)$ achieved partial remission (PR), yielding an overall tumor response rate of $19.5 \% .35$ (45.5\%) patients achieved stable disease (SD), and a further $27(35.1 \%)$ patients showed progressive disease (PD) during treatment. The median survival of the responder group (CR+PR, median 363 days; 95\% CI: 284-705) was significantly longer than that of the non-responder group (SD+PD, median 203 days; 95\% CI: 194-278; $p=0.03, \log$ rank test).

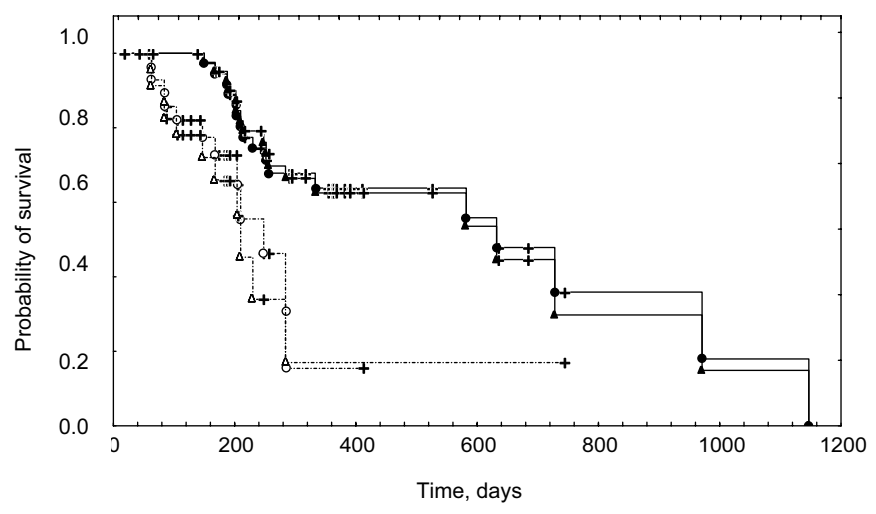

Figure 1. Probability of survival of CA 19-9 responders (solid line) and non-responders (dashed line).

+ : Censored data.

O: according to the criterion by Ishii et al. [21]: CA 19-9 responder: median 295 days (range: 64-1,147; 95\% CI: 285-445 days); CA 19-9 non-responder: median 174 days (range: $17-411$; 95\% CI: 134-198 days) $\mathrm{p}=$ 0.022 (log rank).

$\Delta$ : according to the criterion by Gogas et al. [25]: CA 19-9 responder: median 270 days (range: 64-1,147; 95\% CI: 271-409 days); CA 19-9 non-responder: median 144 days (range: $17-743$; 95\% CI: 111-225 days) $\mathrm{p}=$ 0.017 (log rank).

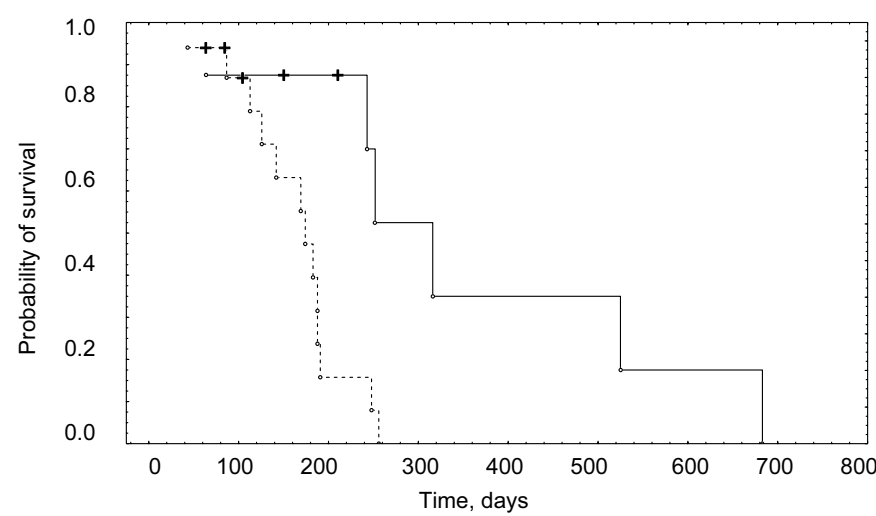

Figure 2. Probability of survival of CA 19-9 responders according to Ishii [21] (solid line) and non-responders (dashed line) of patients with progressive disease by CT imaging criteria $(n=27)$; +: Censored data.

CA 19-9 responder: median 247 days (range: 64-683; 95\% CI: 135-475 days); CA 19-9 non-responder: median 142 days (range: 43-256; 95\% CI: 109-175 days) $\mathrm{p}=0.04$ ( $\log$ rank).

\section{CA 19-9 Serum Concentrations and Response by Radiologic} Imaging

The relationship between serum CA 19-9 changes and response to chemotherapy as evaluated by CT scan is shown in detail in table 2, informations on sensitivity and specificity of CA 19-9 response in relation to CT response are given in table 3. All 4 patients who achieved CR showed a $\geq 50 \%$ decline from baseline in CA 19-9 levels within 2 months of the start of treatment, and were thus considered CA 19-9 responders. Among the 11 patients who achieved PR, 10 were considered CA 19-9 responders. For the 35 patients who achieved SD, 21 
Table 2. CA 19-9 and response according to imaging criteria $(\mathrm{n}=77)$

\begin{tabular}{|c|c|c|c|c|}
\hline & \multicolumn{2}{|c|}{ CT scan responder } & \multicolumn{2}{|c|}{ CT scan non-responder } \\
\hline & Complete response & Partial response & Stable disease & Progressive disease \\
\hline Patients, n & 4 & 11 & 35 & 27 \\
\hline \multicolumn{5}{|l|}{ Baseline CA 19-9 level, U/ml } \\
\hline Median & 1,747 & 2,097 & 486 & 1,911 \\
\hline Range & $134-3,318$ & $51-21,573$ & $53-102,000$ & $44-742,398$ \\
\hline \multicolumn{5}{|l|}{ CA $19-9$ changes, n (\%) } \\
\hline Overall decrease $(\mathrm{n}=58)$ & $4(100)$ & $11(100)$ & $31(89)$ & $12(44)$ \\
\hline Decrease to <32U/ml (n = 11) & $3(75)$ & $3(27)$ & $4(11)$ & $1(4)$ \\
\hline Increase from baseline $(n=19)$ & $0(0)$ & $0(0)$ & $4(11)$ & $15(56)$ \\
\hline \multicolumn{5}{|l|}{ CA $19-9$ response $(\geq 50 \%)^{a}$} \\
\hline CA 19-9 responder $(n=43)$ & $4(100)$ & $10(91)$ & $21(60)$ & $8(30)$ \\
\hline CA $19-9$ non-responder $(\mathrm{n}=34)$ & $0(0)$ & $1(9)$ & $14(40)$ & $19(70)$ \\
\hline \multicolumn{5}{|l|}{ CA $19-9$ response $(\geq 15 \%)^{b}$} \\
\hline CA 19-9 responder $(n=48)$ & $4(100)$ & $10(91)$ & $23(66)$ & $11(41)$ \\
\hline CA $19-9$ non-responder $(\mathrm{n}=29)$ & $0(0)$ & $1(9)$ & $12(34)$ & $16(59)$ \\
\hline
\end{tabular}

a As defined by Ishii et al. [21].

${ }^{\mathrm{b}}$ As defined by Gogas et al. [25]. qualified as CA 19-9 responders, and the CA 19-9 levels for most patients who progressed increased above baseline levels. $(15 / 27)$. However, 12 of the 27 patients in this group showed a biochemical response, characterized by decreases in CA 19-9 levels (8 of them qualified as CA 19-9 responders) despite tumor progression documented by CT scan.

\section{CA 19-9 Response and Survival}

To identify the best model predicting tumor response we evaluated the CA 19-9 response using thresholds of $15 \%$ and $50 \%$. According to the response criteria reported by Ishii et al. [16] (CA 19-9 decrease $\geq 50 \%$ ) we identified 43 patients qualifying as CA 19-9 responders and 34 patients as CA 19-9 non-responders. CA 19-9 responders survived significantly longer than CA 19-9 non-responders $(\mathrm{p}=0.022)$ (fig. 1). The median survival of CA 19-9 responders was 295 days (95\% CI: 285-445), while CA 19-9 non-responders had a survival of 174 days (95\% CI: $134-198 ; p=0.022)$. Using a cut-off for CA 199 response as defined by Gogas et al. [20] (CA 19-9 decrease $\geq 15 \%$ ) we observed 48 CA $19-9$ responders with a median survival of 270 days (95\% CI: 271-409) and 29 non-responders with a median survival of 144 days (95\% CI: 111-225) ( $\mathrm{p}=$ 0.017). As demonstrated in figure 2 , a significant difference of survival was not detected between the two models ( $p>0.05)$. Patients identified as having progressive disease according to CT imaging criteria showed a median survival of 188 days (95\% CI: 109-321 days). Dividing this group into patients who did and did not respond, according to CA 19-9 response, CA 19-9 responders (response criteria by Ishii) had a significantly longer median survival than did CA 19-9 non-responders (247 days (95\% CI: $135-475)$ vs. 142 days (95\% CI: 109-175); p = 0.04) (fig. 2).

\section{Discussion}

In this study, sequential CA 19-9 values were determined in patients with advanced pancreatic cancer treated with a combination of gemcitabine and cisplatin $[6,8]$. The therapeutic efficacy of this combination treatment is supported by a 1-year survival rate of $38 \%$. Burris and coworkes reported a 1-year survival rate of $18 \%$ for patients treated with single-agent gemcitabine, while a significantly lower rate of $2 \%$ was obtained with 5-FU [4].

Reliable parameters of treatment efficacy are necessary tools to guide antitumor treatment. In pancreatic cancer, however, timeliness and reliability of response evaluation are difficult to achieve using conventional imaging procedures. Inclusion of desmoplastic tissue into the baseline tumor volume may cause an underestimation of tumor reduction during therapy, while inclusion of surrounding inflammatory tissue could result in an overestimation of response [3]. Although CT is one of the most reliable modalities for response evaluation, the correlation of CT measurements of tumor volume and that of resected specimens was shown to be limited [16].

In search for an objective and easily obtained endpoint during evaluation of chemotherapeutic efficacy, we prospectively measured CA 19-9 serum concentrations in patients undergo- 
Table 3. CA 19-9

response and $\mathrm{CT}$ findings

\begin{tabular}{ll}
\hline Sensitivity & $93.3 \%$ \\
Specifity & $53.2 \%$ \\
Positive predictive value & $32.5 \%$ \\
Negative predictive value & $97.1 \%$ \\
\hline
\end{tabular}

ing combination chemotherapy. In metastatic disease, the absolute value of pretreatment CA 19-9 levels is not a prognostic factor for survival due to a great interpatient variability [13]. This study, therefore, focused on the analysis of CA 19-9 kinetics rather than absolute values.

For a correct interpretation of CA 19-9 levels, several points have to be taken into consideration:

1. The range of normal CA 19-9 levels is rather broad. Studies in healthy volunteers demonstrated a median CA 19-9 concentration of $3.1 \mathrm{U} / \mathrm{ml}$, while the 10 -fold greater value of $31.9 \mathrm{U} / \mathrm{ml}$ was equivalent to the 95 th percentile.

2. Elevated CA 19-9 serum concentration levels occur in $1-4 \%$ of benign diseases, such as cholecystitis, obstructive jaundice, cholelithiasis, cholangitis, hepatitis, and liver cirrhosis [12].

3. Patients lacking the Lewis-antigen glycosyltransferase (Lewis $^{\mathrm{a}-\mathrm{b}-}$ ) are unable to produce CA 19-9. This deficiency is observed in $7-10 \%$ of the general population, who accordingly will not show CA 19-9 elevations in the course of pancreatic cancer [12].

A previous analysis of Halm and co-workers [19] indicated that CA 19-9 response, namely a CA $19-9$ decrease by $\geq 20 \%$ within 2 months after start of treatment, might be the strongest independent predictor of survival. Based on the response definition of Gogas and co-workers (CA 19-9 decrease by $\geq 15 \%$ ), CA $19-9$ responders of the present study showed a significantly longer survival (270 days; 95\% CI: 271-409) than non-responders (144 days; 95\% CI: 111-225; p = 0.017). By comparison, when the response criteria established by Ishii and coworkers [16] were used (CA 19-9 decrease by $\geq 50 \%$ from baseline) CA 19-9 responders survived for a median of 295 days (95\% CI: 285-445), while non-responders had a survival of 174 days (95\% CI: 134-198; $\mathrm{p}=0.022)$. These results suggest that different cut-offs of CA $19-9$ decrease ( $\geq 15 \%$ vs. $\geq 50 \%$ ) occurring within the same frame of treatment will yield comparable results since the impact on survival using the two models was not significantly different ( $p>0.05$, figure 1 ).

In a further step, CA 19-9 kinetics were compared to response evaluation by radiographic imaging. With one exception, all patients who achieved a remission according to imaging were also CA 19-9 responders. This observation indicates that responses defined by imaging are closely paralleled and supported by CA $19-9$ kinetics. On the other hand, $60 \%$ (21/35) of patients with SD, and $30 \%(8 / 27)$ of patients diagnosed with progression by imaging criteria were categorized as CA 19-9 responders. It appears that treatment effects are more rapidly reflected by changes of biological parameters such as tumor markers, while changes of tumor volume as analyzed by imaging procedures occur at a much slower rate.

Of 27 patients identified as having progressive disease by CT imaging 12 showed a biochemical response with regard to CA 19-9 levels (fig. 2). This observation was not completely unexpected because tumor marker decreases in apparently progressing patients have also been noted by others [16, 19]. The importance of this finding is best demonstrated by an evaluation of survival. Even among patients with progressive disease according to CT evaluation, CA 19-9 responders lived significantly longer than non-responders ( 247 vs. 142 days; $p=0.04$ ). The most probable explanation resides in the known difficulty to adequately assess the tumor size of pancreatic cancers by radiological imaging [16, 19].

In conclusion, CT imaging still remains the gold-standard of response evaluation in advanced and metastatic pancreatic cancer. But it may be concluded that CA 19-9 kinetics are an additional and helpful parameter for evaluating the response and predicting survival in patients undergoing cytotoxic treatment for metastatic pancreatic cancer.

\section{References}

1 Ahlgren JD: Chemotherapy for pancreatic carcinoma. Cancer 1996;78(Suppl 3):654-663.

2 Heinemann V: Gemcitabine - progress in the treatment of pancreatic cancer. Oncology 2001;60:8-18.

3 Brambs HJ, Claussen CD: Pancreatic and ampullary carcinoma. Ultrasound, computed tomography, magnetic resonance imaging and angiography. Endoscopy 1993;25:58-68.

4 Burris HA 3rd, Moore MJ, Andersen J, Green MR, Green MR, Rothenberg ML, Modiano MR, Cripps MC, Portenoy RK, Storniolo AM, Tarassoff P, Nelson R, Dorr FA, Stephens CD, Von Hoff DD: Improvements in survival and clinical benefit with gemcitabine as first-line therapy for patients with advanced pancreas cancer: A randomized trial. J Clin Oncol 1997;15:2403-2413.
5 Brodowicz T, Wolfram RM, Kostler WJ, Tomek S, Vaclavik I, Steger GG, Teleky B, Fugger R, Jakesz $\mathrm{R}$, Zielinski CC: Phase II study of gemcitabine in combination with cisplatin in patients with locally advanced and/or metastatic pancreatic cancer. Anticancer Drugs 2000;11:623-628.

6 Heinemann V, Schermuly MM, Stieber P, Schulz L, Jüngst D, Wilkowski R, Schalhorn A: CA 19-9: A pedictor of response in pancreatic cancer treated with gemcitabine and cisplatin. Anticancer Res 1999;19:1-3.

7 Philip PA, Zalupski MM, Vaitkevicius VK, Arlauskas P, Chaplen R, Heilbrun LK, Adsay V, Weaver D, Shields AF: Phase II study of gemcitabine and cisplatin in the treatment of patients with advanced pancreatic carcinoma. Cancer 2001; 92:569-577.
8 Heinemann V, Wilke H, Mergenthaler HG, Clemens M, König H, Illiger HJ, Arning M, Schalhorn A, Possinger K, Fink U: Gemcitabine and cisplatin in the treatment of advanced or metastatic pancreatic cancer. Ann Oncol 2000;11:1399-1403.

9 Rothenberg ML, Abbruzzese JL, Moore M, Pertenoy RK, Robertson JM, Wanebo HJ: A rationale for expanding the endpoints for clinical trials in advanced pancreatic carcinoma. Cancer 1996;78 (Suppl 3):627-632.

10 Plebani, M, Basso, D, Del Favero, G, Ferrara C, Meggiato T, Fogar P, Mangano F, Ricciardi G, Burlina A: Clinical utility of TPS, TPA and CA 19-9 measurement in pancreatic cancer. Oncology 1993; 50:436-440. 
11 Sakahara H, Endo K, Nakajima K, Nakashima T, Koizumi M, Ohta H, Hidaka A, Kohno S, Nakano Y, Naito A: Serum CA 19-9 concentrations and computed tomography findings in patients with pancreatic carcinoma. Cancer 1986;57:1324-1326.

12 Lamerz R: Role of tumour markers, cytogenetics. Ann Oncol 1999;4(Suppl 10):59-63.

13 Lundin J, Roberts P J, Kuusela P, Haglund C: The prognostic value of preoperative serum levels of CA 19-9 and CEA in patients with pancreatic cancer. Br J Cancer 1994;69:515-519.

14 Sperti C, Pasquali C, Catalini S, Cappellazzo F, Bonadimani B, Behboo R, Pedrazzoli S: CA 19-9 as a prognostic index after resection for pancreatic cancer. J Surg Oncol 1993;52:137-141.

15 Okusaka T, Okada S, Sato T, Wakasugi H, Saisho $\mathrm{H}$, Furuse J, Ishikawa O, Matsuno S, Yokoyama S Tumor markers in evaluating the response to radiotherapy in unresectable pancreatic cancer. Hepatogastroenterology 1998;45:867-872.
16 Ishii H, Okada S, Sato T, Wakasugi H, Saisho H, Furuse J, Ishikawa O, Matsuno S, Yokoyama S: CA 19-9 in evaluating the response to chemotherapy in advanced pancreatic cancer. Hepatogastroenterology 1997:44:279-283.

17 Safi F, Schlosser W, Falkenreck S, Beger HG: Prognostic value of CA 19-9 serum course in pancreatic cancer. Hepatogastroenterology 1998;45:253-259.

18 Stieber P: Möglicher Einsatz der Tumormarker in der Nachsorge. Bayer Int 1996;16:22-31.

19 Halm U, Schumann T, Schiefke I, Witzigmann H, Mössner J, Keim V: Decrease of CA 19-9 during chemotherapy with gemcitabine predicts survival time in patients with advanced pancreatic cancer. Br J Cancer 2000;82:1013-1016.
20 Gogas H, Lofts FJ, Evans TR, Daryanani S, Mansi JL: Are serial measurements of CA 19-9 useful in predicting response to chemotherapy in patients with inoperable adenocarcinoma of the pancreas? Br J Cancer 1998;77:325-328.

21 World Health Organization: WHO Handbook for Reporting Results of Cancer Treatment. Geneva, WHO Offset Publication No. 48, 1979.

22 Ajani JA, Welch SR, Raber MN, Fields WS, Krakoff ICH: Comprehensive criteria for assessing therapy-induced toxicity. Cancer Invest 1990;8:147159.

23 Kaplan EL, Meier P: Nonparametric estimation from incomplete observations. J Am Stat Assoc 1959;53:457-481. 